OPEN ACCESS

Edited by:

Fatima-Zohra Mokrane, Université Toulouse III Paul

Sabatier, France

Reviewed by:

Giovannella Palmieri,

Federico II University Hospital, Italy

Yuchuan $\mathrm{Hu}$,

Tangdu Hospital, China Alexandre Nguyen,

$\mathrm{CHU}$ Toulouse Rangueil, France

*Correspondence:

Junlin Zhou

Izuzil601@163.com

Specialty section:

This article was submitted to

Cancer Imaging and Image-directed Interventions,

a section of the journal

Frontiers in Oncology

Received: 20 November 2020 Accepted: 03 March 2021

Published: 25 March 2021

Citation:

Zhou Q, Ke X, Man J, Zhang B, Wang $F$ and Zhou J (2021) Predicting

Masaoka-Koga Clinical Stage of Thymic Epithelial Tumors Using Preoperative Spectral Computed Tomography Imaging.

Front. Oncol. 11:631649. doi: 10.3389/fonc.2021.631649

\section{Predicting Masaoka-Koga Clinical Stage of Thymic Epithelial Tumors Using Preoperative Spectral Computed Tomography Imaging}

\author{
Qing Zhou ${ }^{1,2,3}$, Xiaoai Ke ${ }^{1,3}$, Jiangwei Man ${ }^{1}$, Bin Zhang ${ }^{1,2,3}$, Furong Wang ${ }^{1}$ \\ and Junlin Zhou ${ }^{1,3 *}$ \\ ${ }^{1}$ Lanzhou University Second Hospital, Lanzhou, China, 2 Second Clinical School, Lanzhou University, Lanzhou, China, \\ ${ }^{3}$ Key Laboratory of Medical Imaging of Gansu Province, Lanzhou, China
}

Objectives: To investigate the utility of spectral computed tomography (CT) parameters for the prediction of the preoperative Masaoka-Koga stage of thymic epithelial tumors (TETs).

Materials and Methods: Fifty-four patients with TETs, aged from 37 to 73 years old, an average age of $55.56 \pm 9.79$ years, were included in the study.According to the MasaokaKoga staging method, there were 19 cases of stage I, 15 cases of stage II, 8 cases of stage III, and 12 cases of stage IV disease. All patients underwent dual-phase enhanced energy spectral CT scans. Regions of interest (ROIs) were defined in sections of the lesion with homogeneous density, the thoracic aorta at the same level as the lesion, the outer fat layer of the lesion, and the anterior chest wall fat layer. The single-energy CT value at 40$140 \mathrm{keV}$, iodine concentration, and energy spectrum curve of all lesion and thoracic aorta were obtained. The energy spectrum CT parameters of the lesions, extracapsular fat of the lesions, and anterior chest wall fat in stage I and stage II were obtained. The energy spectrum CT parameters of the lesions, enlarged lymph nodes and intravascular emboli in the 3 groups were obtained. The slope of the energy spectrum curve and the normalized iodine concentration were calculated.

Results: In stage I lesions, there was a statistically significant difference between the slope of the energy spectrum curve for the lesion and those of the fat outside the lesion and the anterior chest wall in the arteriovenous phase $(P<0.001, P<0.001)$. The energy spectrum curve of the tumor parenchyma was the opposite of that of the extracapsular fat. In stage II lesions, there was a statistically significant difference between the slope of the energy spectrum curve for the anterior chest wall and those of the lesion and the fat outside the lesion in the arteriovenous phase $(P<0.001, P<0.001)$. The energy spectrum curve of the tumor parenchyma was consistent with that of the extracapsular fat. Distinction between stage I and || tumors be evaluated by comparing the energy spectrum curves of the mass and the extracapsular fat of the mass. The accuracy rate of is $79.4 \%$. For stages III and IV, there was no significant difference in the slope of the energy spectrum curve of the tumor parenchyma, metastatic lymph node, and intravascular embolism $(P>0.05)$. The energy 
spectrum curve of the tumor parenchyma was consistent with that of the enlarged lymph nodes and intravascular emboli. The two radiologists have strong consistency in evaluating TETs Masaoka-Koga staging, The Kappa coefficient is 0.873,(95\%Cl:0.7680.978).

Conclusion: Spectral CT parameters, especially the energy spectrum curve and slope, are valuable for preoperative TET and can be used in preoperative staging prediction.

Keywords: thymic epithelial tumor, spectral CT, imaging, Masaoka-Koga clinical staging, predicts

\section{INTRODUCTION}

Thymic epithelial tumors (TETs), which include thymoma and thymic cancer, originate from thymic epithelial cells and are composed of different proportions of epithelial cells and lymphocytes. TETs are the most common tumors in the anterior mediastinum, accounting for $47 \%$ of tumors in this location (1). However, their overall incidence is not high, accounting for $1.3-2.2 / 10^{6}$ cases $(2,3)$. TETs are common in elderly people but rare in children and have been reported in patients aged 10 to 80 years, with an average age of 50 to 60 years among men and women (4). Although some patients present with paraneoplastic autoimmune diseases, most patients have no clinical symptoms. Among these diseases, myasthenia gravis is the most common, affecting approximately one-third of patients. Surgery remains the most common treatment for TETs. Thoroughness of tumor resection is an important factor that influences the prognosis of patients, as the prognosis is significantly better after complete resection than after incomplete resection (5). Tumor diameter, tumor resection style, World Health Organization (WHO) classification, Masaoka-Koga stage, and postoperative radiotherapy and chemotherapy are independent factors that affect the prognosis of patients with thymoma. As a result of the correlation between the CT imaging characteristics of TETs and clinical stage $(6,7)$, tumor stage is evaluated before surgery based on imaging parameters and appropriate treatment strategies are applied. Computed tomography (CT) is currently the preferred method for identifying and characterizing thymic tumors and provides higher sensitivity and specificity (8). The current CT-based staging approach for TETs is based on the appearance of tumor images, and they remain difficult to accurately stage; magnetic resonance imaging (MRI) combined with diffusionweighted imaging and apparent diffusion coefficient values can also be used in quantitative analyses (9). The staging of TETs using MRI is more accurate than that when using CT; however, MRI scanning requires higher, for example, longer inspection time and restrictions on metal foreign bodies in the body. More convenient, non-invasive, and quantifiable indicators are necessary for preoperative staging.

There are many staging systems for TETs (10), and the most commonly used is the Masaoka-Koga staging system. The Masaoka-Koga staging system is based on the tumor invasion range, surrounding structure invasion and implantation, and distant metastasis, among other factors $(8,11,12)$. The latest staging system is the eighth edition of the TNM staging system (13). A recent study (14) collected 217 responses from 37 countries in four continents; $78 \%$ of scholars thought the TNM classification was useful $(\mathrm{N}=169)$, and $87 \%$ of scholars still use the Masaoka-Koga staging system $(\mathrm{N}=189)$. Staging is related to the prognosis of TETs $(9,15)$, and the treatment of different stages of disease are also different. Surgery is generally considered an effective treatment method $(16,17)$; however, some researchers have pointed out that for some patients with thymoma, surgical treatment may not be the best treatment method (18). It has been reported that for patients with stage I thymoma according to the Masaoka-Koga staging, open surgical resection carries the risk of potential surgical damage and other complications, and CT-guided percutaneous radiofrequency treatment of stage I thymoma is related to minor trauma, fewer complications, and good treatment results (19). In patients with Masaoka-Koga stage I and II thymoma, after appropriate surgical resection, auxiliary radiation treatment has no obvious effect on outcomes (20). Platinum-based chemotherapy remains the standard first-line treatment for patients with advanced or metastatic TETs (21).

Based on the correlation between stage and tumor treatment decisions and prognosis, it is necessary to use non-invasive imaging methods to predict the stage before surgery; CT is currently the preferred method for identifying and characterizing thymic tumors, providing higher sensitivity and specificity $(8,22)$. Preoperative CT analysis and understanding of the tumor margin, capsule, surrounding invasion levels, pleural implants, lymph nodes, and distant metastases can predict the stage of TETs and have a positive effect on patient treatment and prognosis (23). However, conventional CT is subjective and is based on characterization of the lesion, multiple spectral CT variables can be used to quantify tumor indicators and more accurate preoperative staging.

\section{MATERIALS AND METHODS}

This study was approved by the institutional review board, and the requirement for written informed consent was waived due to the retrospective nature of this study.

\section{General Information}

Sixty-two patients with surgically and pathologically confirmed TETs treated in our hospital from October 1, 2014 to December 
31, 2020 were enrolled; among these, eight patients with insufficient image quality for regions of interest (ROIs) to be drawn were excluded. Finally, 54 patients were included, comprising 27 men and 27 women aged from 37 to 73 years old, with an average age of $55.56 \pm 9.79$ years. According to the Masaoka-Koga staging system, 19 patients had stage I, 15 patients had stage II, 8 patients had stage III, and 12 patients had stage IV disease. The 54 patients were divided into three groups: non-invasive group (stage I), surrounding fat group (stage II), and surrounding structures and distant metastasis group (stages III and IV). The most common clinical manifestations were intermittent cough, chest tightness, chest pain, and shortness of breath. Eight patients had myasthenia gravis, which manifested as drooping eyelids, limb weakness, and difficulty chewing. Seven of these patients were identified during physical examination, and three patients had recurrence. All patients were treated surgically, and the specimens were sent for pathological examination postoperatively.

\section{Instruments and Methods}

All patients underwent plain and dual-phase-enhanced energy spectrum CT (Discovery CT 750 HD; GE Healthcare, Waukesha, WI, USA). Patients were supine during the scan, which was conducted from the apex to the bottom of the lung. The scanning parameters were as follows: flat scan tube at a voltage of $120 \mathrm{kV}$, enhanced scan tube at a voltage of $80 / 140 \mathrm{kV}$ with fast switching, rack speed of $0.6 \mathrm{~s} / \mathrm{r}$, tube current of $325 \mathrm{~mA}$, pitch of 0.983:1, detector coverage of $40 \mathrm{~mm}$, collimator width of $1.25 \mathrm{~mm}$, and reconstruction layer thickness and layer spacing of $1.25 \mathrm{~mm}$. An iodinated contrast agent (Ultravist 370, Bayer Schering Pharma, Berlin, Germany) was used at a flow rate of 3-4 mL/s and a dose of $1.0 \mathrm{~mL} / \mathrm{kg}$ injected via the anterior cubital vein using a highpressure syringe (Ulrich Medical, Ulm, Germany). The trigger threshold for thoracic aorta monitoring was 80 Hounsfield units. The arterial phase scan was performed $8 \mathrm{~s}$ after the trigger, while the venous phase scan was performed $30 \mathrm{~s}$ after the trigger.

\section{CT Image Analysis}

Two radiologists used the AW 4.6 workstation GSI Viewer software to perform CT scans. ROIs $5 \times 5 \mathrm{~mm}$ in size were delineated in homogeneous lesions while avoiding necrotic cysts, calcification, and vascular shadows. ROIs in the thoracic aorta, the fat layer within $10 \mathrm{~mm}$ outside the lesion capsule, and the fat layer on the anterior chest wall were delineated with $5 \times 5$-mm ROIs. Enlarged lymph nodes and intravascular emboli were outlined by ROIs of the same size. Each ROI was drawn three times to obtain an average value. The single-energy CT value, energy spectrum curve, and iodine concentration in the ROI was recorded. The standardized iodine concentration (NIC) was determined using the $\mathrm{IC}_{\text {lesion }} / \mathrm{IC}$ chest equation, where $\mathrm{IC}_{\text {lesion }}$ is the iodine concentration in the lesion and IC chest is the iodine concentration in the thoracic aorta. The slope was calculated as $\left(\mathrm{CT}_{40}-\mathrm{CT}_{100}\right) /(40-100)$, where $\mathrm{CT}_{40}$ is the $\mathrm{CT}$ value at $40 \mathrm{keV}$ and $\mathrm{CT}_{100}$ is the $\mathrm{CT}$ value at $100 \mathrm{keV}$.

\section{Pathological Images}

Pathological images were first observed under low and high magnification by a pathologist with 3-5 years of work experience. Diagnosis was based on the WHO histological classification. Microscopic observation was performed to assess whether the tumor had invaded the surrounding fat and connective tissue, and whether it had invaded the mediastinal pleura, pericardium, and lungs. It was also clarified whether an intravascular embolus was a thrombus or a tumor, and whether there was metastasis to the lymph nodes. The diagnosis was confirmed by a pathologist with more than 10 years of work experience.

\section{Masaoka-Koga Stage}

In combination with the postoperative pathology, tumor staging was performed based on whether the tumor capsule was intact during surgery, the surrounding fatty layer was clear, and the mediastinal pleura, pericardium, lungs, and blood vessels were adherent and extensively invaded $(8,11,12)$. Stage I tumors were defined as those where the capsule was intact and no extracapsular invasion was observed under the microscope. Stage II tumors were defined as those where macroscopic and microscopic invasion of the mediastinal adipose tissue could be observed, with no invasion of the mediastinal pleura or pericardium. Stage III tumors were defined as those with visible invasion of adjacent structures (such as the pericardium, large blood vessels, or lungs) were observed. Stage IV-A tumors were defined as those with pleural dissemination (pleural or pericardial metastasis) (was observed) and stage IV-B tumors were defined as those with lymph or bloodbased metastasis to a location outside the thoracic region was observed. Based on the extent of tumor invasion, the tumors were divided into a non-invasive group (stage I), invasion of the surrounding fat group (stage II), and invasion of surrounding structures (pleura, pericardium, lung, blood vessels) and distant metastasis group (stages III and IV).We performed TNM staging based on all data and performed a consistency analysis with Masaoka-Koga staging.

\section{Statistical Methods}

SPSS 23.0 software was used for analysis; t-test was used for quantitative comparisons between two groups. Multiple sets of quantitative data were analyzed by one-way analysis of variance, and variances were analyzed using the Kruskal-Wallis test. Pairwise comparisons between groups were performed using the Bonferroni method. The Kappa value was used to evaluate the agreement between two observers. Calculate the accuracy of the energy spectrum CT parameters for TETs staging. $\mathrm{P}<0.05$ indicating that the differences were statistically significant.

\section{RESULTS}

For all 54 cases, there was a strong consistency between TNM staging and Masaoka-Koga staging (ICC=0.852). According to TNM staging, there were more stage I cases than according to the Masaoka-Koga staging. In the two staging methods, there was no significant change in the data for stages III and IV. The two radiologists have strong consistency in evaluating TETs Masaoka-Koga staging, The Kappa coefficient is 0.873 (95\% CI: 0.768-0.978). 


\section{Energy Spectrum CT Results for Three Groups of Lesions}

The single-energy CT values at 40-140 keV, NIC, and slope of the energy curve in the non-invasive group (stage I), surrounding fat group (stage II), and surrounding structures (pleura, pericardium, lung, blood vessels) and distant metastases group (stages III and IV) were not significantly different (Table 1).

\section{CT Results for Stage I and Stage II Lesions, Lesion Extracapsular Fat, and Anterior Chest Wall Fat}

In stage I, the difference in the slope of the energy spectrum curve of tumor parenchyma, tumor extracapsular fat, and anterior chest wall fat was statistically significant $(\mathrm{P}<0.001, \mathrm{P}<0.001)$

(Table 2) (Figure 1). In stage II, the difference between the tumor parenchyma, the fat outside the tumor, and the slope of the spectrum curve of the anterior chest wall was statistically significant $(\mathrm{P}<0.001, \mathrm{P}<0.001)$ (Table 3) (Figure 2). According to the slope of the energy spectrum curve of the outer fat layer of the lesion, combined with the energy spectrum curve graph, the accuracy of spectral CT for predicting the Masaoka-Koga stage I and II lesions was $79.4 \%$.

\section{CT Results for Stage IV Lesions, Intravascular Tumor Thrombi, and Metastatic Lymph Nodes}

There were no significant differences in the slopes of the energy spectrum curves for stage IV lesions, metastatic lymph nodes, and intravascular emboli $(\mathrm{P}>0.05)$ (Table 4) (Figures 3 and 4). The slope of the energy spectrum curve combined with the energy spectrum curve graph, the accuracy of assessing lymph node metastasis and embolic properties is $80.0 \%$.

\section{DISCUSSION}

Three parameters were evaluated in this study, the single energy CT value of the lesion, the NIC, and the slope of the energy spectrum curve. The single-energy imaging mode of energy spectrum CT simulates the image of an object in the presence of a monochromatic X-ray source. The attenuation of different tissues changes in accordance with changes in the X-ray beam energy. A lower single-energy beam can increase the density resolution of the image, which helps to show lesions. The slope of the energy spectrum curve reflects the magnitude of the enhancement. The larger the slope is, the greater the magnitude of the enhancement will be, and the energy spectrum curve of the tissue changes greatly at low energy. Therefore, low energy (40-100 keV) was selected to determine the slope of the energy spectrum curve for each group. The shape-like energy spectrum curve may reflect the homology of the region of interest. The iodine (water) concentration of the lesion was directly obtained from the material separation map for the energy spectrum CT, which quantitatively reflects the iodine uptake and distribution in the lesion. To eliminate individual differences, the NIC was used to compare the iodine (water) base values. The NIC was more reliable than the iodine concentration (24). NIC is an indirect reflection of the iodine content of a lesion, that is, the degree of strengthening.

The 54 patients evaluated in this study included 19 with stage I, 15 with stage II, 8 with stage III lesions, and 12 with stage IV lesions according to the Masaoka-Koga staging. The 54 patients were divided into three groups: non-invasive group (stage I),

TABLE 1 | Spectral CT multi-parameter results of the three groups in stages (Arterial phase).

\begin{tabular}{|c|c|c|c|c|c|}
\hline Parameters & $\begin{array}{l}\text { Non-invasive group } \\
\text { (stage I) }\end{array}$ & $\begin{array}{l}\text { Invasion of the surrounding fat group } \\
\text { (stage II) }\end{array}$ & $\begin{array}{l}\text { Invasion of surrounding structures and distant metastasis } \\
\text { groups (stage III和IV) }\end{array}$ & $\mathbf{F} / \mathrm{H}$ & $\mathbf{P}$ \\
\hline $40 \mathrm{keV}$ & $179.51 \pm 48.56$ & $177.28 \pm 43.52$ & $169.24 \pm 39.12$ & 0.291 & 0.749 \\
\hline $50 \mathrm{keV}$ & $130.22 \pm 31.71$ & $129.98 \pm 28.93$ & $123.04 \pm 25.68$ & 0.373 & 0.691 \\
\hline $60 \mathrm{keV}$ & $98.86 \pm 21.42$ & $99.53 \pm 21.25$ & $92.15 \pm 18.06$ & 0.744 & 0.481 \\
\hline $70 \mathrm{keV}$ & $79.87 \pm 16.32$ & $80.90 \pm 15.92$ & $74.10 \pm 13.39$ & 1.044 & 0.360 \\
\hline $80 \mathrm{keV}$ & $68.60 \pm 14.05$ & $70.30 \pm 12.96$ & $64.67 \pm 10.92$ & 0.892 & 0.416 \\
\hline $90 \mathrm{keV}$ & $61.11 \pm 12.61$ & $63.63 \pm 11.55$ & $58.23 \pm 10.35$ & 0.889 & 0.418 \\
\hline $100 \mathrm{keV}$ & $55.56 \pm 12.00$ & $58.28 \pm 10.84$ & $53.06 \pm 9.81$ & 0.912 & 0.408 \\
\hline $110 \mathrm{keV}$ & $51.65 \pm 11.76$ & $54.49 \pm 10.44$ & $49.38 \pm 9.59$ & 0.915 & 0.408 \\
\hline $120 \mathrm{keV}$ & $48.89 \pm 11.71$ & $51.88 \pm 10.23$ & $46.80 \pm 9.51$ & 0.916 & 0.407 \\
\hline $130 \mathrm{keV}$ & $46.77 \pm 11.72$ & $49.85 \pm 10.15$ & $44.81 \pm 9.48$ & 0.906 & 0.411 \\
\hline $140 \mathrm{keV}$ & $45.13 \pm 11.75$ & $48.26 \pm 10.07$ & $43.27 \pm 9.49$ & 0.891 & 0.417 \\
\hline $\mathrm{NIC}$ & $0.15 \pm 0.05$ & $0.12 \pm 0.04$ & $0.13 \pm 0.04$ & 1.191 & 0.313 \\
\hline Curve slope & $-2.06 \pm 0.74$ & $-1.98 \pm 0.63$ & $-1.93 \pm 0.58$ & 0.191 & 0.827 \\
\hline
\end{tabular}

TABLE 2 | Slopes of energy spectrum curves, extracapsular fat and anterior chest wall fat of TETs stage I.

\begin{tabular}{|c|c|c|c|c|c|}
\hline & $\begin{array}{c}\text { Slope of the energy spectrum } \\
\text { curve of the lesion }\end{array}$ & $\begin{array}{l}\text { Slope of energy spectrum curve of } \\
\text { extracapsular fat in lesions }\end{array}$ & $\begin{array}{l}\text { Slope of energy spectrum curve of } \\
\text { anterior chest wall fat }\end{array}$ & F/H & $P$ \\
\hline Arterial phase & $-2.00 \pm 0.76$ & $1.94 \pm 0.69^{*}$ & $2.32 \pm 0.33$ & 293.52 & 0.000 \\
\hline Venous phase & $-2.10 \pm 0.51$ & $1.72 \pm 0.48^{\star}$ & $2.08 \pm 0.33$ & 500.89 & 0.000 \\
\hline
\end{tabular}

Compared with the slope of the lesion energy spectrum curve, ${ }^{*} P<0.05$; Compared with the slope of the anterior chest wall energy spectrum curve, ${ }^{\#} P<0.05$. 


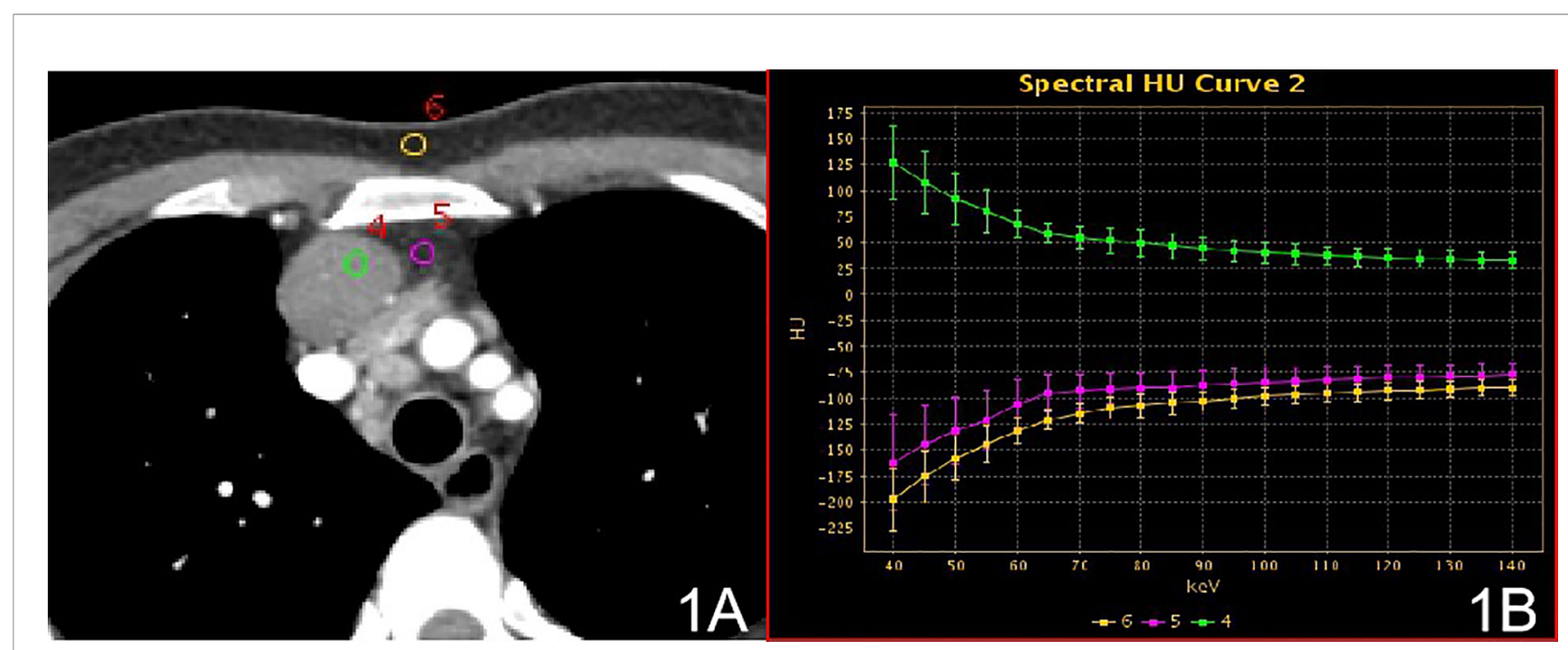

FIGURE 1 | Stage I, (A) $70 \mathrm{keV}$ single-energy CT value of the arterial phase is used to outline the ROI of the lesion; (B) spectrum of arterial lesions (green), spectrum of fat outside the lesion (purple) and spectrum of anterior chest wall fat (yellow).

TABLE 3 | Slopes of energy spectrum curves of, extracapsular fat and anterior chest wall fat TETs stage II.

Slope of the energy spectrum curve of the lesion Slope of energy spectrum curve of extracapsular fat in lesions

\begin{tabular}{ccc}
$\begin{array}{c}\text { Slope of energy spectrum curve } \\
\text { of anterior chest wall fat }\end{array}$ & F/H & P \\
\hline $2.18 \pm 0.27$ & 223.54 & 0.000 \\
$2.05 \pm 0.28$ & 409.71 & 0.000 \\
\hline
\end{tabular}

\begin{tabular}{|c|c|c|c|c|c|}
\hline Arterial phase & $-1.91 \pm 0.66$ & $-1.27 \pm 0.57^{\star}$ & $2.18 \pm 0.27$ & 223.54 & 0.000 \\
\hline Venous phase & $-2.27 \pm 0.48$ & $-1.46 \pm 0.53^{\star} \#$ & $2.05 \pm 0.28$ & 409.71 & 0.000 \\
\hline
\end{tabular}

Compared with the slope of the lesion energy spectrum curve, ${ }^{*} P<0.05$; Compared with the slope of the anterior chest wall energy spectrum curve, ${ }^{\#} P<0.05$.

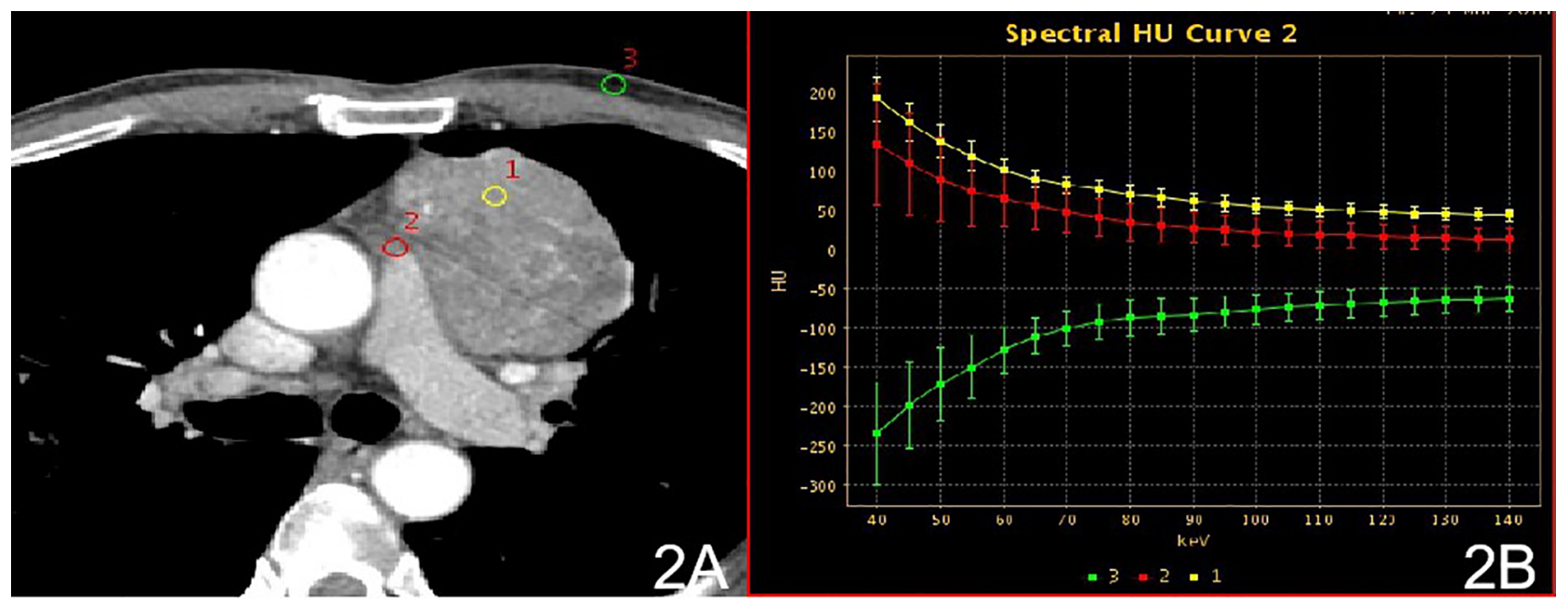

FIGURE 2 | Stage II, (A) $70 \mathrm{keV}$ single-energy CT value of the arterial phase is used to outline the ROI of the lesion; (B) arterial lesion spectrum (yellow), extra-focal fat spectrum (red) and anterior chest wall fat spectrum (green).

invasive surrounding fat group (stage II), and invasive surrounding structures (pleura, pericardium, lung, blood vessels) and distant metastasis group (stages III and IV). In the qualitative diagnosis, irregular or lobed edges lesions on conventional CT. It may indicate that the tumor capsule and extracapsular fat have been invaded (25). In this study, ROIs were delineated in stage I and stage II solid lesions, extracapsular fat, and anterior chest wall fat. The energy spectrum curves for 
TABLE 4 | Slope analysis of spectrum curves of lesions, metastatic lymph nodes, and intravascular emboli in group 3 (stages III and IV).

Slope of the energy spectrum curve of the lesion Slope of the energy spectrum curve Slope of the energy spectrum curve $\mathrm{F} / \mathrm{H} \quad \mathrm{P}$ of metastatic lymph nodes of intravascular emboli

\begin{tabular}{|c|c|c|c|c|}
\hline Arterial phase & $-1.83 \pm 0.64$ & $-1.53 \pm 0.68$ & $-1.45 \pm 0.65$ & $0.964 \quad 0.393$ \\
\hline Venous & $-1.72 \pm 0.59$ & $-1.69 \pm 0.57$ & $-1.37 \pm 0.77$ & 2.4820 .101 \\
\hline
\end{tabular}

phase
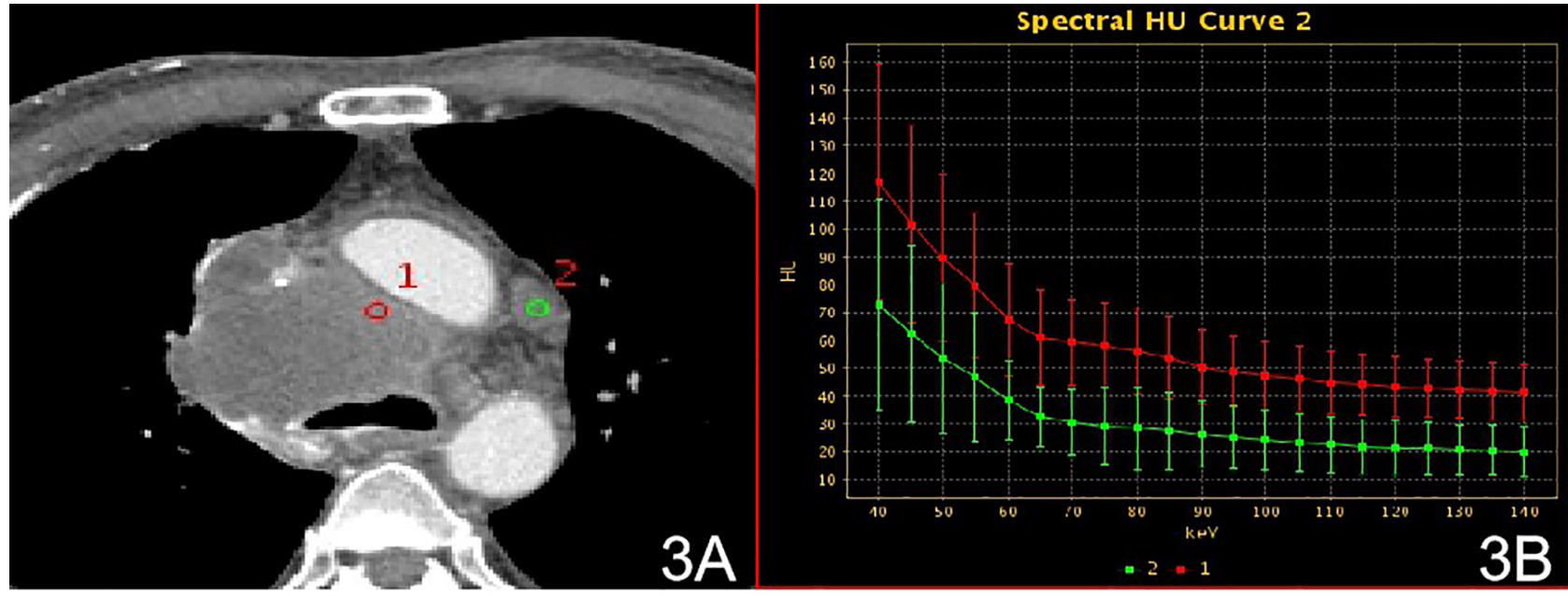

FIGURE 3 | Stage IV, (A) $70 \mathrm{keV}$ single-energy CT value of the arterial phase is used to outline the ROI of the lesion; (B) arterial lesion spectrum (red), lymph node spectrum (green)
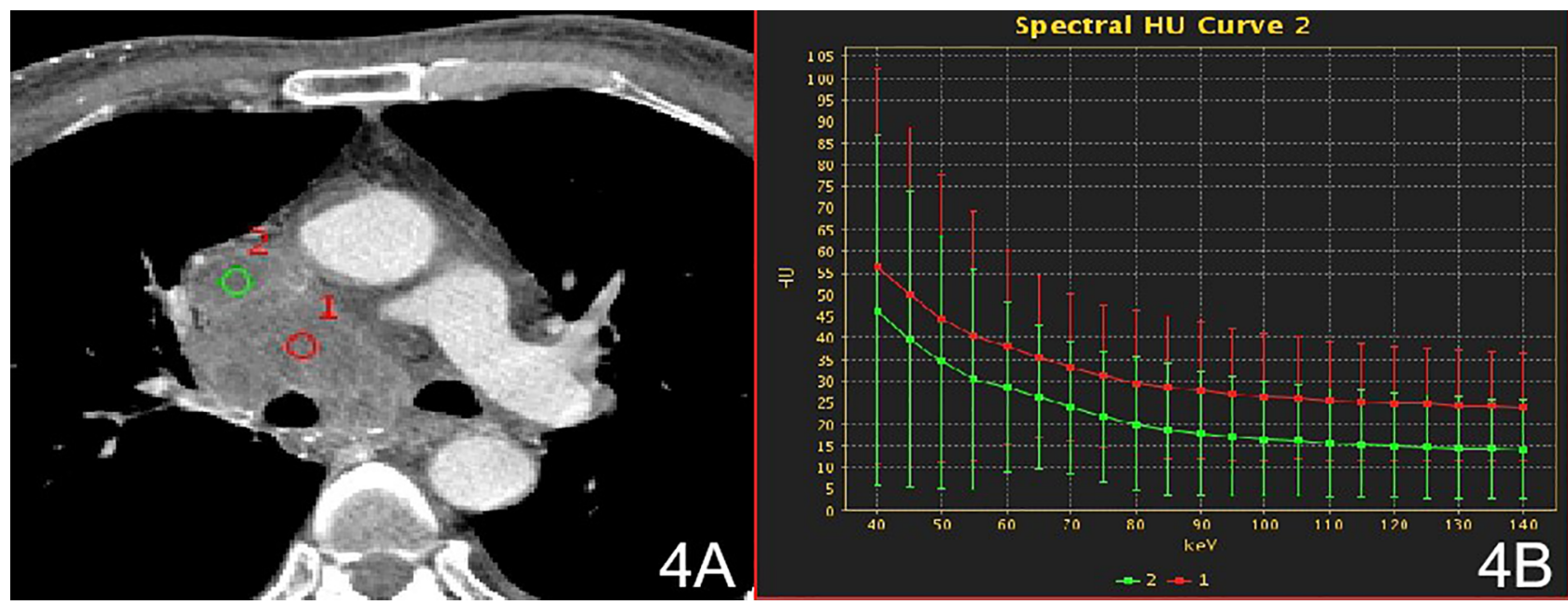

FIGURE 4 | Stage IV, (A) $70 \mathrm{keV}$ single-energy CT value of the arterial phase is used to outline the ROI of the lesion; (B) Arterial lesion spectrum (red), intravascular emboli spectrum (green).

the lesion, extracapsular fat, and anterior chest wall fat were obtained. In stage I lesions, the energy spectrum curve of the tumor parenchyma and the energy spectrum curve of the extracapsular fat have the opposite shape, and the energy spectrum curve of the extracapsular fat and the front chest wall fat have the same shape, indicating that the lesion did not extend outside the capsule. The slope of the spectrum curve for the anterior chest wall fat in stage II lesions showed statistically 
significant differences with those of the spectra for the lesion and the lesion's extracapsular fat, indicating that the lesion had invaded the extracapsular fat. According to the spectrum curve, the lesion and the fat in the outer capsule of the lesion were homologous. In this study, ROIs were delineated in pathologically confirmed mediastinal lymph node with metastasis, intravascular tumor emboli, and solid lesions. The spectral curves for the metastatic lymph nodes, intravascular emboli, and lesions were consistent and the differences in the slopes were not statistically significant, indicating that the lesions were homologous to lymph nodes and intravascular emboli (26). Among these parameters, the energy spectrum curve objectively reflects whether the tumor parenchyma and extracapsular fat are homologous, and whether the enlarged lymph nodes and emboli are metastatic.

In conclusion, distinction between stage I and II tumors may be evaluated by comparing the energy spectrum curves of the mass and the extracapsular fat of the mass. Areas of interest should be outlined in suspicious enlarged lymph nodes and intravascular emboli before surgery to determine whether the lymph nodes have metastasized and the nature of the emboli in the vessel.

\section{Limitations}

The limitations of our study are as follows: This study was a retrospective and single-center study, lacking multi-center data verification and the sample size was small. The accuracy of preoperative predicting staging needs further large sample size research. Measurement of the ROI of fat outside the capsule stays a difficult points, even though we used the average value of 3 ROIs of the same size drawn in the fat layer $10 \mathrm{~mm}$ outside the capsule of the lesion to reflect as much as possible whether the lesion has extracapsular fat invasion.

\section{REFERENCES}

1. Chen JL, Weisbrod GL, Herman SJ. Computed tomography and pathologic correlations of thymic lesions. J Thorac Imaging (1988) 3(1):61-5. doi: 10.1097/00005382-198801000-00010

2. Scorsetti M, Leo F, Trama A, D'Angelillo R, Serpico D, Macerelli M, et al. Thymoma and thymic carcinomas. Crit Rev Oncol Hematol (2016) 99:332-50. doi: 10.1016/j.critrevonc.2016.01.012

3. Engels EA. Epidemiology of thymoma and associated malignancies. J Thorac Oncol (2010) 5(10 Suppl 4):S260-5. doi: 10.1097/JTO.0b013e3181f1f62d

4. den Bakker MA, Roden AC, Marx A, Marino M. Histologic classification of thymoma: a practical guide for routine cases. J Thorac Oncol (2014) 9(9 Suppl 2): S125-30. doi: 10.1097/JTO.0000000000000297

5. Banna GL, Sheel A, Sheel V, Bille A, Routledge T, Fernando S, et al. Treatment and prognostic factors of patients with thymic epithelial tumors at first recurrence or progression. Future Oncol (2017) 13(27):2429-39. doi: 10.2217/ fon-2017-0236

6. Iannarelli A, Sacconi B, Tomei F, Anile M, Longo F, Bezzi M, et al. Analysis of $\mathrm{CT}$ features and quantitative texture analysis in patients with thymic tumors: correlation with grading and staging. Radiol Med (2018) 123(5):345-50. doi: 10.1007/s11547-017-0845-4

7. Qu YJ, Liu GB, Shi HS, Liao MY, Yang GF, Tian ZX. Preoperative CT findings of thymoma are correlated with postoperative Masaoka clinical stage. Acad Radiol (2013) 20(1):66-72. doi: 10.1016/j.acra.2012.08.002

8. Masaoka A, Monden Y, Nakahara K, Tanioka T. Follow-up study of thymomas with special reference to their clinical stages. Cancer (1981) 48

\section{DATA AVAILABILITY STATEMENT}

All data generated or analyzed during this study are included in this published article.

\section{AUTHOR CONTRIBUTIONS}

QZ designed the study, performed the literature search and data analysis, and drafted the manuscript. JZ and QZ participated in the conception and design of the study, performed the quality assessment and helped to revise the manuscript. JM, FW, BZ and $\mathrm{XK}$ performed the study selection and statistical analysis and helped to draft the manuscript. QZ, JM, XK, BZ, and FW performed the data extraction and analysis, modified the language. All authors contributed to the article and approved the submitted version.

\section{FUNDING}

This work was supported the National Natural Science Foundations of China(81772006).

\section{ACKNOWLEDGMENTS}

Thanks to all the partners who contributed to this research, including Qing Zhou, Jiangwei Man, Furong Wang, Xiaoai Ke, Junlin Zhou. Special thanks to Ms. Furong Wang from the Pathology Department and Mr. Jiangwei Man from the Department of Surgery.

(11):2485-92. doi: 10.1002/1097-0142(19811201)48:11<2485::AIDCNCR2820481123>3.0.CO;2-R

9. Kong LY, Zhang W, Zhou Y, Xu H, Shi HB, Feng Q, et al. Histogram analysis of apparent diffusion coefficient maps for assessing thymic epithelial tumours: correlation with world health organization classification and clinical staging. Br J Radiol 2018 (1084) 91:20170580. doi: 10.1259/bjr.20170580

10. Weissferdt A, Moran CA. Staging of thymic epithelial neoplasms: thymoma and thymic carcinoma. Pathol Res Pract (2015) 211(1):2-11. doi: 10.1016/ j.prp.2014.06.007

11. Koga K, Matsuno Y, Noguchi M, Mukai K, Asamura H, Goya T, et al. A review of 79 thymomas: modification of staging system and reappraisal of conventional division into invasive and non-invasive thymoma. Pathol Int (1994) 44(5):359-67. doi: 10.1111/j.1440-1827.1994.tb02936.x

12. Detterbeck FC, Nicholson AG, Kondo K, Van Schil P, Moran C. The Masaoka-Koga stage classification for thymic malignancies: clarification and definition of terms. J Thorac Oncol (2011) 6(7 Suppl 3):S1710-6. doi: 10.1097/ JTO.0b013e31821e8cff

13. Detterbeck FC, Stratton K, Giroux D, Asamura H, Crowley J, Falkson C, et al. The IASLC/ITMIG Thymic Epithelial Tumors Staging Project: proposal for an evidence-based stage classification system for the forthcoming (8th) edition of the TNM classification of malignant tumors. J Thorac Oncol (2014) 9:S65-72. doi: 10.1097/JTO.0000000000000290

14. Ruffini E, Fang W, Guerrera F, Huang J, Okumura M, Kim DK, et al. The IASLC Thymic Tumors Staging Project. The Impact of the 8th Edition of the UICC/AJCC TNM Stage Classification of Thymic Tumors: Results of a Survey. J Thorac Oncol (2019) 15(3):436-47. doi: 10.1016/j.jtho.2019.11.013 
15. Tassi V, Vannucci J, Ceccarelli S, Gili A, Matricardi A, Avenia N, et al. Stagerelated outcome for thymic epithelial tumours. BMC Surg (2019) 18(Suppl 1):114. doi: 10.1186/s12893-018-0434-z

16. Detterbeck F, Youssef S, Ruffini E, Okumura M. A review of prognostic factors in thymic malignancies. J Thorac Oncol (2011) 6(7 Suppl 3):S1698-704. doi: 10.1097/JTO.0b013e31821e7b12

17. Wright CD, Wain JC, Wong DR, Donahue DM, Gaissert HA, Grillo HC, et al. Predictors of recurrence in thymic tumors: importance of invasion, World Health Organization histology, and size. J Thorac Cardiovasc Surg (2005) 130 (5):1413-21. doi: 10.1016/j.jtcvs.2005.07.026

18. Koppitz H, Rockstroh JK, Schüller H, Standop J, Skowasch D, Müller-Hermelink $\mathrm{HK}$, et al. State-of-the-art classification and multimodality treatment of malignant thymoma. Cancer Treat Rev (2012) 38(5):540-8. doi: 10.1016/j.ctrv.2011.11.010

19. Dong J, Yuan S, Chang B, Huang J, Geng X, Cai X, et al. An exploratory study of CT-guided percutaneous radiofrequency ablation for stage I thymoma. Cancer Imaging (2019) 19(1):80. doi: 10.1186/s40644-019-0267-8

20. Gielda BT, Peng R, Coleman JL, Thomas CR, Cameron RB. Treatment of early stage thymic tumors: surgery and radiation therapy. Curr Treat Options Oncol (2008) 9(4-6):259-68. doi: 10.1007/s11864-008-0080-2

21. Conforti F, Pala L, Giaccone G, De Pas T. Thymic epithelial tumors: From biology to treatment. Cancer Treat Rev (2020) 86:102014. doi: 10.1016/j.ctrv.2020.102014

22. Zhao Y, Chen H, Shi J, Fan L, Hu D, Zhao H. The correlation of morphological features of chest computed tomographic scans with clinical characteristics of thymoma. Eur J Cardiothorac Surg (2015) 48(5):698-704. doi: $10.1093 /$ ejcts/ezu475
23. Ottlakan A, Borda B, Morvay Z, Maraz A, Furak J. The Effect of Diagnostic Imaging on Surgical Treatment Planning in Diseases of the Thymus. Contrast Media Mol Imaging (2017) 2017:9307292. doi: 10.1155/2017/ 9307292

24. Vogel J, Lin L, Litzky LA, Berman AT, Simone CB 2nd. Predicted Rate of Secondary Malignancies Following Adjuvant Proton Versus Photon Radiation Therapy for Thymoma. Int J Radiat Oncol Biol Phys (2017) 99(2):427-33. doi: 10.1016/j.ijrobp.2017.04.022

25. Jung KJ, Lee KS, Hun J, Kim J, Kim TS, Kim EA, et al. Malignant thymic epithelial tumors: CT.pathologic correlation. AJR Am J Roentgenol (2001) 176:433-9. doi: 10.2214/ajr.176.2.1760433

26. Aran S, Daftari Besheli L, Karcaaltincaba M, Gupta R, Flores EJ, Abujudeh HH. Applications of dual-energy CT in emergency radiology. AJR Am J Roentgenol (2014) 202:W314-24. doi: 10.2214/AJR.13.11682

Conflict of Interest: The authors declare that the research was conducted in the absence of any commercial or financial relationships that could be construed as a potential conflict of interest.

Copyright (c) 2021 Zhou, Ke, Man, Zhang, Wang and Zhou. This is an open-access article distributed under the terms of the Creative Commons Attribution License (CC BY). The use, distribution or reproduction in other forums is permitted, provided the original author(s) and the copyright owner(s) are credited and that the original publication in this journal is cited, in accordance with accepted academic practice. No use, distribution or reproduction is permitted which does not comply with these terms 